\title{
TENDENCIAS DE INUESTIGACIÓN EN LAS CIENCIAS DE LA SALUD SOBRE LA SALUD MENTAL Y SU ORIENTACIÓN COMUNITARIA
}

\author{
José Camargo \\ John Pedraza, Amanda Ortiz, Luciana Restrepo, Carlos Cortés $\$ \S \S$
}

\begin{abstract}
RESUMEN
Se revisaron las tendencias internacionales de investigación en ciencias de la salud sobre la salud mental y su enfoque comunitario en la última década a través de bases de datos disponibles, para posteriormente agrupar los resultados en categorías que emergieron de la revisión. Se encontró que existe un gran interés por este campo de investigación, pero las investigaciones siguen estando mayoritariamente direccionadas a la relación existente entre salud y enfermedad siendo pocos los proyectos que centran su atención en los aspectos positivos y comunitarios de la salud. De lo anterior se desprende la necesidad de fortalecer las investigaciones en aspectos relacionados con la salud mental en comunidad y los efectos positivos de la salud y su influencia en la calidad de vida.
\end{abstract}

Palabras Claves: Salud mental, salud mental comunitaria, tendencias, investigación.

\section{INTRODUCCIÓN}

El presente artículo tiene como objetivo revisar y analizar las tendencias de investigación en ciencias de la salud a nivel internacional sobre la salud mental y su orientación hacia la comunidad en la última década, para lo cual se realizó la revisión de la literatura científica en bases de datos, teniendo como criterio de inclusión en la búsqueda las palabras claves salud mental, salud mental comunitaria, tendencias e investigación. El resultado de la búsqueda se agrupó en categorías emergentes en la revisión para posteriormente realizar el análisis.

La investigación en salud es un tema prioritario en el Informe de Salud Mental de 2013 de la Organización Mundial de la Salud OMS (1), donde afirma que el tema debe estar presente en los ámbitos nacional e internacional y exige, por parte de todos los países, mayor atención para poder identificar las diferentes problemáticas susceptibles de ser investigadas y poder desde el ámbito científico generar estrategias que permitan mejorar la cobertura sanitaria a nivel mundial y, por ende, la salud de la población.

Entre los temas prioritarios en salud podemos encontrar el de la salud mental, definido por la OMS como un "estado de bienestar en el cual el individuo es consciente de sus propias capacidades, puede afrontar las tensiones normales de la vida, puede trabajar de forma productiva y fructífera y es capaz de hacer una contribución a su comunidad" (2) y considerado central en las políticas de salud pública en la actualidad.

Llama la atención que Salaverry (3) muestra cómo el interés y la evolución del concepto y las estrategias de intervención sobre la salud mental no son recientes y que su origen puede rastrearse en dos corrientes diferentes; por un lado, las interpretaciones sobrenaturales de la enfermedad mental presentes ya en las culturas antiguas que atribuían la enfermedad mental a dioses o demonios y, por otro lado, la Medicina occidental naciente en la antigua Grecia en el siglo V a. C. en donde es concebida de forma naturalista y explicada desde las teorías del humor y, su evolución posterior hasta la

§§ Estudiantes de Maestría en Salud Mental Comunitaria. Universidad El Bosque - japedrazap@gmail.com

Carta Comunitaria. Vol. 24. Número 137. Marzo - Abril 2016 
actualidad en las diferentes concepciones teóricas fruto de la investigación científica. Esta doble línea de interés por el tema llevó a que por mucho tiempo la salud mental estuviese fluctuando en la sociedad entre lo público y lo privado, lo que retrasó su incorporación como problema de salud pública.

Según Restrepo (4) la incorporación de la salud mental en la salud pública es un tema prioritario a nivel profesional, académico y político, confirmado por los diferentes estudios epidemiológicos que muestran las contribuciones realizadas por los trastornos mentales a la carga de enfermedades a nivel mundial. Sin embargo, presenta algunas dificultades de orden epistemológico y conceptual que no son fáciles de resolver y condicionan la forma de investigar e intervenir en el ámbito de la salud.

En los últimos años, (Sineke) Ten Horn (5) las investigaciones en salud mental han venido cambiando, pasando de un enfoque hospitalario a uno comunitario, lo cual ha derivado en un cambio en las tendencias de investigación en este campo, prestando mayor atención a la prevención, la cronicidad de las enfermedades, la colaboración en salud mental, la atención primaria y el entorno del sujeto de estudio como factor importante en la salud.

En América Latina este cambio de enfoque, según Caldas de Almeida (6), recibió un fuerte impulso tras la Declaración de Caracas en 1990, en la cual se aboga por una mayor atención a la salud mental de los pueblos y por la superación del modelo de atención hospitalario y el desarrollo de nuevos modelos de atención basados en la comunidad, sin embargo, existen dificultades actuales en el cumplimiento de estas metas.

Teniendo en cuenta estos cambios a nivel mundial en el interés y las tendencias de investigación en la salud mental en los últimos años, a continuación se mencionarán algunas de las publicaciones científicas realizadas en la última década a nivel mundial, para que sirvan como marco de referencia a la hora de determinar posibles áreas de investigación. Son presentadas por categorías temáticas construidas teniendo como referente los temas comunes en los diferentes artículos de investigación revisados, que no pretendieron ser una búsqueda exhaustiva por estar limitada por las bases de datos consultadas.

\section{Salud mental y enfermedad}

Desde hace años se viene argumentando la importancia de la prevención de los trastornos mentales y la promoción de la salud, según lo evidencian algunos autores como Jané (7), quien afirma que las intervenciones en prevención y promoción son eficaces y se puede evidenciar sus alcances a nivel de la salud en la población, basando sus afirmaciones en la eficacia demostrada de diversos programas analizados aplicando técnicas de meta-análisis y recomendando seguir mejorando la eficacia y efectividad de dichas intervenciones, haciendo énfasis en el papel tan importante que cumplen los investigadores para la consecución de dichos logros. De ahí la importancia de la investigación en este campo tan fructífero en la actualidad.

Obiols (8) detectó en un estudio que las problemáticas en el ámbito de la salud mental de mujeres madres con VIH/Sida debido a la falta de atención apropiada en los servicios públicos de salud, afecta directamente sus relaciones interpersonales con quienes establecen vínculos afectivos, entre ellos sus hijos, su pareja y sus familiares, presentando además malestar subjetivo y dificultades en la continuación de los tratamientos médico farmacológicos.

Por su parte, Bello et al (9) han estudiado la prevalencia de la depresión en población adulta en México, encontrando que la población de mujeres es la que más presenta depresión en comparación con los hombres y la prevalencia incrementa con la edad pero disminuye cuando existe mayor grado de escolaridad. En relación con los hombres es de destacar que existe la presencia de mayor depresión en aquellos que viven en zonas rurales.

Conscientes de los problemas de salud asociados al ejercicio profesional en el área de la saludenfermedad, Utrera et al (10) se interesaron en la relación del terapeuta con el paciente y realizaron 
una investigación cuasiexperimental con la finalidad de validar un programa formativo en inteligencia emocional dirigido a las enfermeras que trabajan con pacientes diagnosticados con trastorno límite de personalidad (TLP) donde se analizaban los efectos del programa, y así mejorar la relación entre el profesional de la salud y dicha población de pacientes.

Desde otra perspectiva, Itzhak et al (11) realizaron una investigación sobre diferentes estudios publicados en América Latina y el Caribe entre 1980 y 2004, sobre estadísticas epidemiológicas, encontrando que trastornos como la esquizofrenia tenían una prevalencia de $1 \%$, la depresión mayor $4,9 \%$ y el abuso o la dependencia del alcohol 5,7\%, estableciendo que un gran número de estas personas no recibieron ningún tipo de tratamiento psiquiátrico.

Olfson et al (12), evaluando las tendencias a nivel longitudinal en la población de niños, adolescentes y adultos sobre la salud mental, encontraron que en la última década han venido aumentando los diagnósticos de trastornos mentales y la demanda de atención domiciliaria principalmente en los jóvenes.

Sobre la psicosis Kelleher et al (13) analizaron las experiencias psicóticas de jóvenes relacionadas con una amplia gama de trastornos psicóticos. Encontraron que aquellos con mayores tasas de experiencias psicóticas reportaban un funcionamiento más pobre a nivel social y mayor riesgo de conductas suicidas.

En otro estudio, Zashikhina (14) al evaluar la calidad de vida de adolescentes con enfermedades físicas crónicas como diabetes, asma y epilepsia en Rusia, encontraron evidencia de que su calidad de vida y su salud mental se ve afectada por la gravedad de la enfermedad que cursan.

Por otro lado, en un estudio que vinculó a población de dos países Stickley et al (15) estudiaron la variable soledad y su relación con la salud mental de adolescentes de Rusia y Estados Unidos, encontrando asociación entre la soledad y el aumento de consumo de sustancias psicoactivas en los adolescentes de ambos países, en tanto que no se halló relación con el comportamiento violento; por otro lado, solo se estableció relación entre soledad y salud mental con riesgo de embarazo en la población Rusa.

Hervás et al (16) evaluaron la calidad de vida relacionada con la salud en pacientes con diabetes mellitus tipo 2, determinando que esta población presenta puntuaciones inferiores que la población en general a nivel de salud física, dolor crónico, funciones sociales y rol emocional, lo cual deja en evidencia su percepción de baja calidad de vida.

Finalmente, Caqueo et al (17) describieron la relación existente entre calidad de vida y funcionamiento familiar de pacientes con esquizofrenia en una comunidad latinoamericana, encontrando que, por lo menos esta comunidad a diferencia de otras analizadas en estudios anteriores, presenta un nivel adecuado de calidad de vida relacionada con una fuerte percepción de funcionalidad familiar. Sin embargo, dicha funcionalidad percibida es diferente entre el paciente y su cuidador.

\section{Salud mental y educación}

En el campo de la educación, si bien no se encontraron muchas investigaciones, también se han venido realizando estudios sobre la salud mental en los diferentes niveles educativos, encontrándose estudios como los de Estévez et al (18) que muestran cómo el contexto escolar de los adolescentes está relacionado con su salud mental y confirman los resultados de otras investigaciones al evidenciar la relación existente entre los contextos familiares y escolares en el ajuste psicológico de los jóvenes, lo que los lleva a concluir que los problemas de comunicación en la familia pueden traer consigo dificultades en el ajuste escolar de los adolescentes lo que les acarreará problemas de salud mental.

Micin (19) evaluando la incidencia de psicopatologías y antecedentes de conducta suicida en universitarios, encontró que diferentes trastornos como la depresión, desordenes de personalidad, trastornos adaptativos y del estado de ánimo se encuentran presentes de forma significativa en esta 
población de estudiantes lo que encuentra un alto grado de concordancia con otros estudios realizados a nivel internacional.

Por otro lado, Feldman et al (20) han estado investigando la relación existente entre las variables estrés académico, apoyo social, salud mental y rendimiento académico, en estudiantes universitarios en Venezuela, encontrando que las condiciones positivas de salud mental están relacionadas con un mayor apoyo social y un menor estrés académico. El rendimiento académico disminuye cuando el estrés académico es mayor que el apoyo social.

\section{Salud mental y género}

A continuación se presentan algunas de las investigaciones que se han realizado recientemente y que tienen como uno de sus objetivos principales realizar sus análisis en relación al género y las diferentes problemática de salud mental en diferentes contextos.

Montero et al (21) muestran la importancia de la investigación relacionada con género y salud mental y sus implicaciones e importancia a nivel profesional y en la prestación de los servicios en salud. Enfatizan en la necesidad del trabajo interdisciplinario que tenga en cuenta los elementos biológicos, ambientales y psicosociales implícitos en el bienestar de las personas.

Larrañaga et al (22) compararon la salud física-mental y la calidad de vida en población de cuidadores y no cuidadores haciendo énfasis en sus diferencias de género. En los resultados se puede evidenciar que tuvieron más bajas puntuaciones en calidad de vida y salud física y mental las mujeres cuidadoras que las no cuidadoras. Por otro lado, las cuidadoras presentan más riesgo que los cuidadores de sufrir por sedentarismo, falta de sueño y peor salud mental. Como conclusión de su estudio se puede evidenciar que el cuidador tiene más riesgo a nivel de salud que los no cuidadores, pero son las mujeres cuidadoras las más afectadas.

En relación a los predictores de salud, Sánchez et al (23) abordaron las variables ansiedad, autoestima y satisfacción autopercibida, enfatizando en las diferencias de género entre hombres y mujeres, encontrando que las mujeres presentan más problemas de salud que los hombres; sin embargo, cuando se observan estas diferencias en relación a las condiciones laborales llegan incluso a desaparecer.

\section{Salud mental comunitaria}

Respecto a temas de investigación centrados en lo comunitario, son escasas las publicaciones que se pueden encontrar que aborden el tema comunidad-salud de forma específica, ya que aunque existen investigaciones que abordan los temas de salud mental en población perteneciente a la comunidad, solo una analiza, de alguna manera, las comunidades en torno a la salud mental.

En esta investigación, Bang (24), desde una perspectiva comunitaria, analizó los efectos que tiene la articulación de las estrategias de promoción de la salud mental comunitaria con las diferentes prácticas comunitarias de participación como el arte, la creatividad y el juego en espacio público, evidenciando la dificultad existente en las comunidades para considerar a la población como actores potenciales de participación y gestores de canales efectivos para la participación comunitaria.

\section{Salud mental en contexto laboral y económico}

Al indagar sobre las relaciones entre salud mental y el trabajo y su subsiguiente vínculo con la capacidad económica de las personas, se encuentran diversos estudios publicados que abordan estas temáticas. Uno de ellos es el de Vuori et al (25), quienes han trabajado a nivel de psicología organizacional investigando experimentalmente los efectos de un programa para la creación de recursos que mejoren la salud mental, el desempeño de las actividades laborales y aseguren la permanencia de los empleados en la empresa. Este programa mostró efectos positivos en la salud 
mental de los trabajadores, especialmente aquellos que presentaban sintomatología de depresión o agotamiento laboral y disminuyó las intenciones de retirarse de la institución donde laboran.

Bones et al (26) investigaron la prevalencia de problemas de salud mental en las personas y su relación con variables socioeconómicas, de trabajo y salud en España. Encontraron que la prevalencia de problemas de salud mental es mayor en mujeres $(24,6 \%)$ y las variables más relacionadas con problemas de salud son la separación o divorcio, ser emigrante, presentar más dificultades socioeconómicas que el promedio, tener enfermedades crónicas, estar desempleado, entre otras.

Por otro lado Trucco (27) muestra cómo los problemas de salud mental son comunes en la población y su estrecha relación con el estrés, causando frecuentemente incapacidades, ausentismo laboral y reducción en los niveles de productividad de los empleados en la empresa. Termina mostrando cómo el trabajo es una fuente de promoción de la salud, pero, de igual forma, puede convertirse en un factor de riesgo.

Desde una perspectiva más conceptual, Durmêt et al (28) muestran los principales enfoques teóricos que pretenden explicar la interrelación de la salud mental con el trabajo, entre los que encontramos aquellos modelos con enfoque del desgaste que pretenden encontrar la relación existente entre los componentes biopsicosociales y el desgaste propio de las actividades laborales con las consecuencias para la salud; el de las condiciones generales de vida y trabajo, que aborda los elementos propios de las condiciones de vida y laborales con la salud mental; el del stress como factor que influye en la calidad de vida y la salud; el de la ergonomía que se enfoca en los elementos psicosociales como eje de análisis de la salud-enfermedad $y$, el de la psicopatología del trabajo, relacionado con los componentes psicopatológicos derivados del contexto laboral de las personas.

\section{Salud mental y bienestar}

Una categoría, de gran importancia pero poco explorada, es la estrecha relación existente entre salud mental y bienestar percibido; al respecto se observó que la mayoría de investigaciones consultadas suelen centrarse en los aspectos negativos y patológicos de la salud y que son pocos los que abogan por los componentes positivos de la misma. No obstante, Oramas et al (29) dejan en evidencia que no todas las investigaciones en salud están enfocadas en determinar los aspectos negativos de la salud mental y evidencian desde una perspectiva positiva en la psicología cómo el bienestar psicológico es una variable mediadora entre la persona y su entorno, actuando como un elemento protector e indicador de una buena salud mental.

Desde esta perspectiva Marrero (30) evaluó en adultos diferentes componentes del bienestar, la satisfacción vital, las emociones positivas y negativas, el ajuste psicológico y el optimismo. Encontraron resultados que muestran que la variable sociodemográfica y la salud no presentan relación con el nivel de optimismo de las personas. Las personas optimistas están más ajustadas psicológicamente $y$, por otro lado, las personas que perciben mayor apoyo por parte de los demás se sienten más satisfechas con su vida y sus parejas.

Whetsell y et al (31) estudiaron los niveles de bienestar espiritual y fortaleza relacionándolos con la salud en el adulto mayor y encontraron que a mayor bienestar espiritual existe una mejor salud. En este estudio las mujeres mostraron un mayor nivel de bienestar espiritual que los hombres.

\section{Algunas reflexiones en torno a la salud mental}

Múltiples investigadores han estado reflexionando en la última década sobre la salud mental y su orientación hacia lo comunitario desde muy diversas perspectivas y disciplinas, lo que ha dejado un terreno fértil de oportunidades para las nuevas tendencias de investigación y políticas públicas de regulación y mejoramiento del acceso a la salud dejando en evidencia las limitaciones y vacíos que en este campo de acción existen. Se presentan a continuación algunos de los aportes que estos 
profesionales en torno a la discusión de la salud mental han plasmado y a los cuales se pudo acceder en las bases de datos consultadas.

Marrero (32) muestra cómo en la isla de Tenerife, a pesar de que la atención en salud mental está enfocada en lo comunitario, no se cuenta en la actualidad con sistemas de atención comunitaria apropiados ni con los recursos, servicios y programas para poder cubrir las necesidades en torno a la salud mental.

En este mismo contexto Díaz (33) pone en evidencia cómo la labor de las enfermeras que se encuentran trabajando en las unidades de salud mental comunitaria en Tenerife se ve diezmada cuando no se cuentan con los recursos económicos y de personal apropiados para ejecutar las actividades de atención en salud frente a la alta demanda de consultas que todos los días debe ser atendida.

Frente a las políticas públicas, Desviat (34) enfatiza en la necesidad de preguntarse sobre dichas políticas públicas y sus tendencias a futuro y la demanda de las personas sobre salud mental, antes de pensar en las perspectivas a futuro en psicología y psiquiatría en este campo.

Minoletti (35), luego de realizar un recorrido por la implementación del Plan Nacional de Salud Mental de Chile en un período de 10 años, llega a la conclusión de que los programas e intervenciones llevados a cabo han permitido reducir los problemas de salud mental en la población y aumentado el grado de satisfacción y calidad de vida de las personas.

Un aporte importante al debate es el propuesto por Moral (36) desde la psicología social de la salud a la visión dominante de salud-enfermedad, en la cual se termina solo medicando, lo cual trae más problemas que soluciones a la salud y calidad de vida de las personas. Adicionalmente esto conlleva a la separación del síntoma de su estado psicológico y contexto biográfico y personal del paciente lo que terminaría en una falsificación del problema reduciéndolo todo a una simple sintomatología carente de cualquier sentido personal.

Para terminar, Mebarak et al (37) después de analizar el estado del arte en psicología sobre cómo se entiende el concepto de salud mental, principalmente desde la psicología de la salud, han concluido que dicho concepto implica el desarrollo de estilos de vida y diferentes características personales, interpersonales, sociales y laborales que se enfoquen hacia una visión integradora del bienestar biopsicosocial.

\section{CONCLUSIONES}

Del presente trabajo de revisión de artículos publicados en diferentes bases de datos se puede llegar a las siguientes conclusiones:

Existe un número cada vez mayor de investigaciones que en la actualidad abordan el tema de la salud mental y sus efectos en la población en general; sin embargo, gran parte de estas investigaciones están centradas en problemáticas relacionadas con patologías y enfermedades.

Existen muy pocas investigaciones enfocadas a valorar los aspectos comunitarios de la salud mental, como también escasean aquellas que pretenden valorar los aspectos positivos desencadenados por el bienestar y otras variables en la salud mental.

Se evidencia que existe un gran campo de investigación no explorado en torno a lo comunitario y positivo de la salud que puede ser generador de futuras líneas investigativas que promuevan la salud mental comunitaria.

\section{REFERENCIAS}

(1) Organización Mundial de la Salud. Informe Sobre la salud en el mundo 2013: Investigaciones para una cobertura sanitaria universal. [Internet]. Luxembourg; OMS. 2013; Disponible desde: http://www.who.int/whr/2013/report/es/

(2) Organización Mundial de la Salud. Salud mental: un estado de bienestar. [Internet]. OMS; 2013 Dic. Disponible desde http://www.who.int/features/factfiles/mental_health/es/ 
(3) Salaverry O. La piedra de la locura: inicios históricos de la salud mental. 2012; 9(1): 143-148.

(4) Restrepo D, Jaramillo J. Concepciones de salud mental en el campo de la salud pública. 2012; 30 (2): $202-211$.

(5) (Sineke) Ten Horn G, Nuevas tendencias en la investigación en salud mental. 2004; 90: 167-176.

(6) Caldas de Almeida J. Implementación de políticas y planes de Salud Mental en América Latina. 2007; 71(2): 111-116.

(7) Jané E. La eficacia de la promoción de la salud mental y la prevención de los trastornos mentales. 2004; (89): 67-77.

(8) Obiols M, Stolkiner A. Importancia de la inclusión de la salud mental en la atención integral de mujeres que viven con VIH/Sida. 2012 Nov; XXIII (45): 61-80.

(9) Bello M, Puentes-Rosas E, Medina-Mora M, Lozano R. Prevalencia y diagnóstico de depresión en población adulta en México. 2005; 47(1): 4-11.

(10) Utrera E, Pérez L, Lopera M, Rosado M. Efectividad de una intervención sobre inteligencia emocional en enfermeras especialistas en salud mental. 2014; 11(71): 1-15.

(11) Itzhak R, Caldas J, Vicente B, Andrade L, Caraveo-Anduaga J, Saxena S, Saraceno B. Los trastornos mentales en américa latina y el caribe: asunto prioritario para la salud pública. 2005; 18(4/5): 229-240.

(12) Olfson M, Blanco C, Wang S, Laje G, Correll C. National Trends in the Mental Health Care of Children, Adolescents, and Adults by Office-Based Physicians. 2014; 71(1): 81-90.

(13) Kelleher I, Devlin N, Wigman J, Kehoe A, Murtagh A, Fitzpatrick C, Cannon M. Psychotic experiences in a mental health clinic sample: implications for suicidality, multimorbidity and functioning. $2014 ; 44: 1615-1624$.

(14) Zashikhina A, Hagglof B. Health-related quality of life in adolescents with chronic physical illness in northern Russia: a cross-sectional study. 2014; 12: 1-8.

(15) Stickley A, Koyanagi A, Koposov R, Schwab-Stone M, Ruchkin V. Loneliness and health risk behaviours among Russian and U.S. adolescents: a cross-sectional study. 2014; 14: 1-12.

(16) Hervás A, zabaleta A, De Miguel G, Beldarrain O, Díez J. Calidad de vida relacionada con la salud en pacientes con diabetes mellitus tipo 2. 2007; 30 (1): 45-52.

(17) Caqueo A, Lemos S. Calidad de vida y funcionamiento familiar de pacientes con esquizofrenia en una comunidad latinoamericana. 2008; 20(4): 577-582.

(18) Estévez E, Musitu G, Herrero J. El rol de la comunicación familiar y del ajuste escolar en la salud mental del adolescente. 2005; 28(4): 81-89.

(19) Micin S, Bagladi V. Salud Mental en Estudiantes Universitarios: Incidencia de Psicopatología y Antecedentes de Conducta Suicida en Población que Acude a un Servicio de Salud Estudiantil. 2011; 29(1): 53-64.

(20) Feldman L, Goncalves L, Puignau G, Zaragoza J, Bagés N, De Pablo J. Relaciones entre estrés académico, apoyo social, salud mental y rendimiento académico en estudiantes universitarios venezolanos. 2008; 7(3): 739-751.

(21) Montero I, Aparicio D, Gómez M, MorenoB, Reneses B, Usallf J, Vázquez J. Género y salud mental en un mundo cambiante. $2004 ; 18(1): 175-181$.

(22) Larrañaga I, Martín U, Bacigalipe A, Begiristáin J, Valderrama M, Arregi B. Impacto del cuidado informal en la salud y la calidad de vida de las personas cuidadoras: análisis de las desigualdades de género. $2008 ; 22$ (5): $443-450$.

(23) Sánchez M, Aparicio M, Dresch V. Ansiedad, autoestima y satisfacción autopercibida como predictores de la salud: diferencias entre hombres y mujeres. 2006; 18(3): 584-590.

(24) Bang C, Stolkiner A. Aportes para pensar la participación comunitaria en salud/salud mental desde la perspectiva de redes. 2013; XXIV(46): 123-143.

(25) Vuori J, Toppinen S, Mutanen P. Effects of resource-building group intervention on career management and mental health in work organizations: Randomized controlled field trial. 2012; 97(2): 273-286.

(26) Bones K, Pérez K, Rodríguez M, Borrell C, Obiols J. Prevalencia de problemas de salud mental y su asociación con variables socioeconómicas, de trabajo y salud: Resultados de la Encuesta Nacional de Salud de España. 2010; 22(3):389-395.

(27) Trucco M. El Estrés y la Salud Mental en el Trabajo; Documento de Trabajo de la Asociación Chilena de Seguridad. 2004; 6(14): $185-188$.

(28) Dumêt J, Melo C, Gusmão M, Fernandes J, Guimarães A. Salud mental y trabajo: significados y límites de modelos teóricos. $2006 ; 14(5)$.

(29) Oramas A, Santana S, Vergara A. El bienestar psicológico, un indicador positivo de la salud mental. 2006; 7(1-2): 34-39.

(30) Marrero R, Carballeira M. El papel del optimismo y del apoyo social en el bienestar subjetivo. 2010; 33: 39-46.

(31) Whetsell M, Frederickson K, Aguilera P, Maya J. Niveles de bienestar espiritual y de fortaleza relacionados con la salud en adultos mayores. 2005; 5(1): 72-85.

(32) Marrero D. Realidad de la salud mental en el área norte de Tenerife. $2009 ; 3(3): 25-34$.

(33) Díaz D, González N. La enfermería comunitaria desde la unidad de salud mental comunitaria LA VERAICOD. 2009; 3(3): 51-56.

(34) Desviat M. De lo público, lo privado y el futuro de la asistencia a la salud mental. 2009; 14: 91-98.

(35) Minoletti A, Zaccarias A. Plan nacional de salud mental en chile: 10 años de experiencia. 2005; 18(4-5): 346-358.

(36) Moral M. Crítica a la visión dominante de salud-enfermedad desde la psicología social de la salud. 2008; $94: 85-104$.

(37) Mebarak M, De Castro A, Salamanca M, Quintero M. Salud mental: un abordaje desde la perspectiva actual de la psicología de la salud. 2009; 23: 83-112.

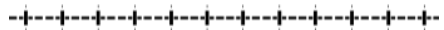

Carta Comunitaria. Vol. 24. Número 137. Marzo - Abril 2016 